\title{
Caring for breast cancer survivors in primary care
}

\author{
Kathryn Trotter, DNP, CNM, FNP-BC, FAANP; April Stouder, MHS, PA-C
}

\begin{abstract}
Women with a history of breast cancer compose the largest group of cancer survivors. Physician assistants and nurse practitioners can play a key role in caring for cancer survivors in primary care settings. This article provides a brief overview and synthesis of current breast cancer guidelines, other resources, and clinical observations that may help primary care providers to translate plans developed by oncology specialists into primary care delivery.

Keywords: breast cancer, survivor, primary care, physical examination, cardiac toxicity, lymphedema
\end{abstract}

\section{Learning objectives}

- Describe the characteristics of breast cancer patients in the United States, breast cancer epidemiology, and current guidelines for ongoing care.

- Discuss the issues surrounding surveillance for breast cancer recurrence and managing long-term and late adverse reactions to cancer treatments.

A fter skin cancer, breast cancer is the most prevalent cancer diagnosed among women in the United States, with an incidence rate of more than 230,000 new cases annually. ${ }^{1}$ One out of every eight American women (12.3\%) will be diagnosed with breast cancer during her lifetime. ${ }^{1}$ Due to increased availability in the United States of early-detection methods as well as improvements in surgical, medical, and radiation therapies, the 5 -year survivorship for all stages combined approaches $90 \%$, and the 10 -year rate is $83 \% .^{1}$ As such, women with a history of breast cancer comprise the largest group of cancer survivors. ${ }^{2}$ About 3.5 million women in the United States have survived breast cancer, including those who have completed treatment as well as those undergoing treatment. ${ }^{2}$ Because the risk of developing breast cancer increases with age, these num-

Kathryn Trotter is an assistant professor at Duke University's School of Nursing in Durham, N.C. April Stouder is an assistant professor in the PA program at Duke University. The authors have disclosed no potential conflicts of interest, financial or otherwise.

DOI:10.1097/01.JAA.0000496950.95334.86

Copyright (C) 2016 American Academy of Physician Assistants

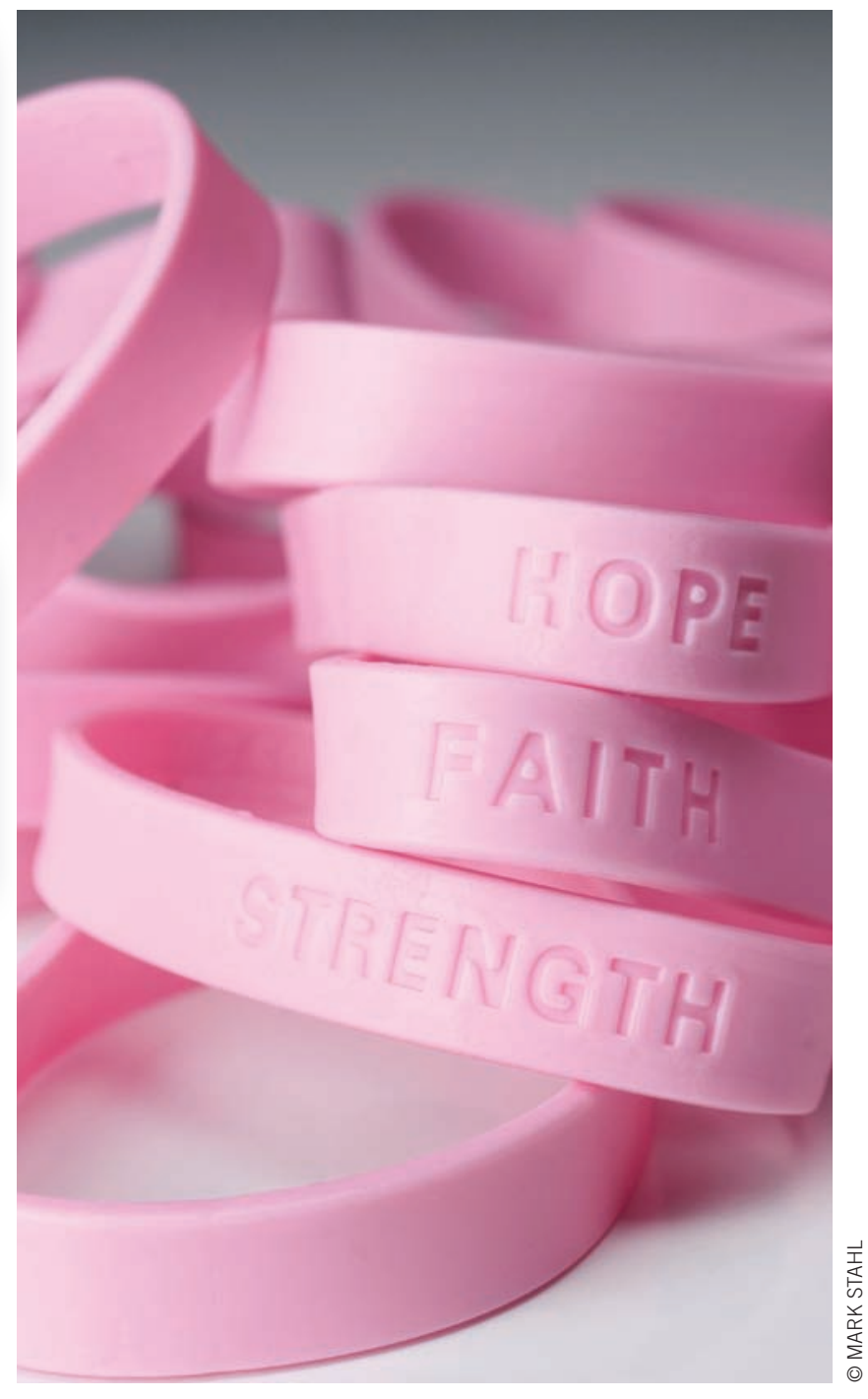

bers are projected to increase due to an aging population. Most women are diagnosed with early-stage disease and more than $80 \%$ will become long-term survivors, living 5 or more years from diagnosis. ${ }^{2}$

After the initial acute phase of treatment, which may include surgery, chemotherapy, radiation therapy, and/or endocrine therapy initiation, survivorship care becomes the focus. This care has historically occurred in specialty settings, but is increasingly moving to primary care due to the pressures of increased patient volume and projected shortages of oncologists. ${ }^{3,4}$ 


\section{Key points}

Women with a history of breast cancer compose the largest group of cancer survivors.

- Long-term follow-up care for breast cancer survivors is increasingly shifting to primary care settings, where physician assistants and NPs may be responsible for patient care.

- Surveillance for recurrence and managing long-term and late adverse reaction to cancer treatments are the mainstay of survivorship care.

Physician assistants and NPs play a key role in caring for cancer survivors in both specialty and primary care settings. Primary care teams caring for cancer survivors have resulted in the same health outcomes as specialist follow-up, with good patient satisfaction. ${ }^{5}$ Furthermore, primary care clinicians may be able to provide more complete preventive follow-up care for some women, with procedures such as Pap smears and vaccinations, than can specialized oncology practices. ${ }^{6}$

This article provides a brief overview and synthesis of current breast cancer guidelines, other resources, and clinical observations that may help primary care providers translate plans developed by oncology specialists into evidence-based follow-up care for breast cancer survivors.

\section{SURVEILLANCE AND SCREENING}

To detect early signs of a recurrence, cancer survivors have historically been subjected to numerous imaging and laboratory tests. This practice led to unnecessary biopsies and further follow-up interventions, as well as increased worry and anxiety, without improvement in mortality. The National Comprehensive Cancer Network (NCCN) and American Society of Clinical Oncology (ASCO) now recommend three basic surveillance methods for breast cancer survivors without metastatic disease (Stage IV) who have reached the 1-year postacute or primary therapy phase following diagnosis:

- a thorough history and physical examination including careful breast examination, performed every 3 to 6 months for the first 3 years after primary treatment, every 6 to 12 months in years 4 and 5 , and then annually

- an annual diagnostic mammogram.

Breast ultrasound or MRI may be indicated with new findings. ${ }^{7-9}$

Serial chest radiographs, bone scans, CT scans, and serum tumor markers are no longer considered standard of care. ${ }^{7,8}$ Furthermore, ASCO recommends that all women perform monthly breast self-awareness examinations. ${ }^{8}$ Women at high risk for familial breast cancer syndromes should receive genetic counseling and may also need more active surveillance, such as breast MRI. ${ }^{10}$

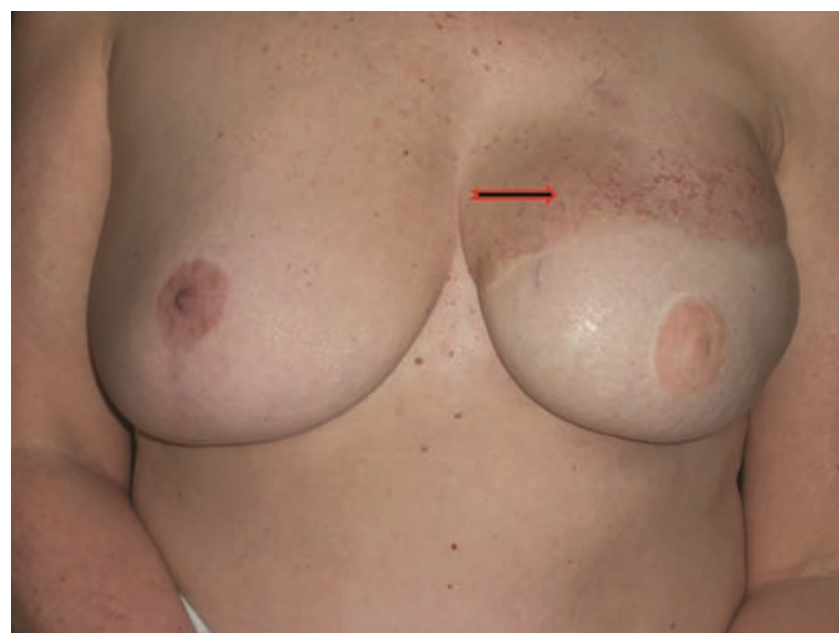

FIGURE 1. Left modified radical mastectomy with reconstruction via transverse rectus abdominis myocutaneous procedure (TRAM flap) with areola and nipple tattoo. Note the left upper breast telangiectasia (arrow) and right reduction mammoplasty incision at 6 o'clock.

Red flags Clinicians should be alert for potential breast cancer recurrence, which typically occurs in the same breast, chest wall, or distantly in the brain, bones, lungs, or liver. Of particular concern are a new breast, axillary, or chest wall mass; new skin lesion over the surgical scar; new-onset headaches; new or worsening deep bony pain (not joint pain); unexplained weight loss; right upper quadrant abdominal pain; jaundice; persistent nausea or dizziness; chest pain; shortness of breath; or persistent cough.

Diagnostic testing may include (per NCCN guidelines): breast imaging via mammogram or ultrasound; complete blood cell count; liver function tests including alkaline phosphatase; CT of the chest, abdomen, and pelvis; brain MRI if the patient has suspicious central nervous system symptoms; bone scan or radiographs of symptomatic bones; or sodium fluoride positron emission test/CT. ${ }^{7}$ Tissue biopsy is essential to document recurrence of disease. Refer the patient back to the specialist if recurrence or new cancer is identified. Patients who have relocated or are not planning to return to their original treating surgeon or oncologist should be referred to local cancer specialists who will request the previous history and treatment summary.

Breast examination Radiation treatment and surgery (including axillary node dissection) alters breast tissue. ${ }^{11}$ During examination, carefully inspect the exposed chest with the patient seated as well as supine, and thoroughly palpate the remaining breast tissue or chest wall. Remaining breast tissue may become firmer due to postradiation fibrosis or surgical scarring, and skin in the radiation field may remain hyperpigmented long after treatment ends (Figures 1-3). Occasionally, benign seroma formation occurs postoperatively, particularly after mastectomy and 


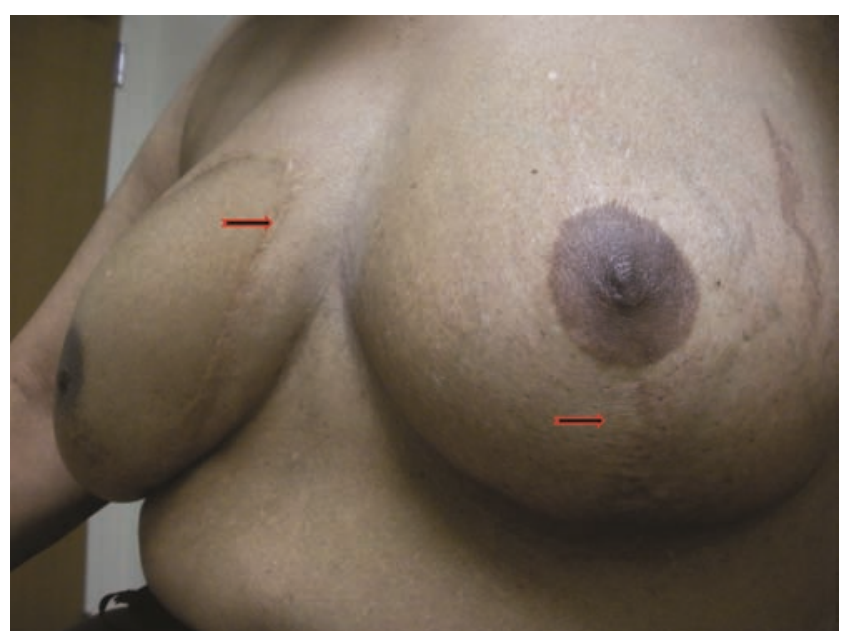

FIGURE 2. Right TRAM flap (arrow) post modified radical mastectomy, with left reduction mammoplasty incision at 6 o'clock (arrow). Scattered striae seen in left breast.

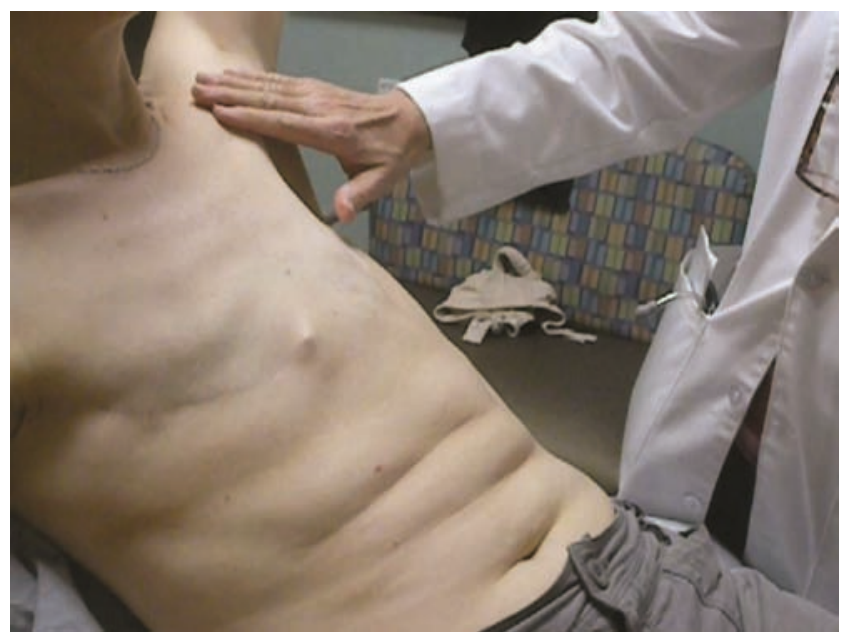

FIGURE 3. Examination post mastectomy (without reconstruction) using chest wall sweep with patient in supine position. Use light-to-moderate touch, covering entire area from clavicle to breast. Skin of right mastectomy incision has slight pucker at medial end.

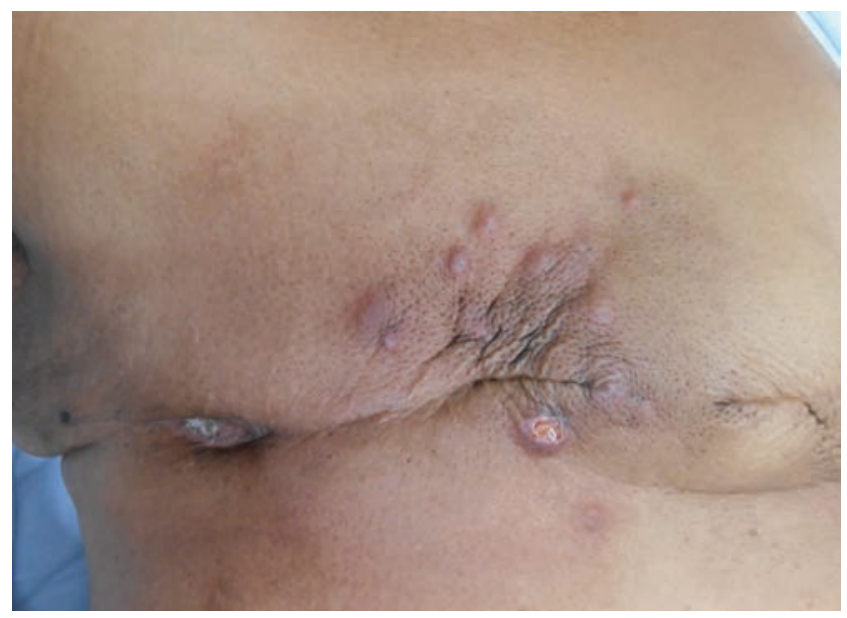

FIGURE 4. Recurrent disease near mastectomy incision. axillary surgery. Percutaneous aspiration may be helpful. ${ }^{12}$ If a new breast lesion is found (Figure 4 ) in a reconstructed breast with or without an implant, follow the same principles of evaluation as with natural breast tissue: palpate, imaging, and biopsy if warranted. Mammogram remains the gold standard for imaging, then ultrasound or breast MRI if the lesion is not viewed on mammogram. Needle biopsy follows, taking care to avoid any implant; occasionally excisional biopsy is done by a surgeon if it is thought the needle would likely hit the implant. For more examination tips, see Table 1.

\section{ADVERSE REACTIONS TO TREATMENT}

Surgery, chemotherapy, and radiation therapy have toxic effects on healthy tissue as well as malignant cells. Both history and physical examination should focus on longterm and late adverse reactions to cancer treatments. Depending on the specific treatments used, breast cancer survivors may be at increased risk for treatment-related effects that can linger for months or years after the acute effects of treatment subside, including cardiac toxicity, peripheral neuropathy, menopausal symptoms, and cognitive changes. ${ }^{13}$

Late effects, that is, new health issues that emerge years after treatment has ended, may include secondary malignancies, decreased bone mineral density, lymphedema, fertility issues, and cardiovascular disease. ${ }^{14}$ These issues can have a major effect on quality of life, functional status, emotional well-being, and interpersonal relationships. ${ }^{13-15}$

Cardiac toxicity Women who have been treated with anthracycline chemotherapy (doxorubicin or epirubicin), trastuzumab, or aromatase inhibitors are at risk for cardiac toxicity, as are those who received irradiation to the left breast/chest wall before 1985, when targeted therapy was less discrete. ${ }^{14}$ The effects of radiation and anthracyclines may have a latent period of 10 to 20 years. ${ }^{16}$ Thus, auscultation of heart sounds is a standard part of the annual examination, though routine ECGs are not warranted. Effects of cancer treatment may include temporary or permanent reduction in left ventricular ejection fraction (temporary reduction is more likely with trastuzumab), valvular disease caused by radiation, and heart failure or coronary artery disease caused by aromatase inhibitors or radiation. Patients who present with signs or symptoms of decreased cardiac function, such as shortness of breath, persistent cough, or tibial edema, should have an ECG and echocardiogram and may need a cardiology consultation.

Peripheral neuropathy Specific chemotherapeutic agents such as taxanes (paclitaxel and docetaxel) can cause peripheral neuropathy, depending on the cumulative dose of the drug. ${ }^{17}$ Additional patient-related risk factors include advanced age, preexisting diabetes, and peripheral nerve dysfunction. Symptoms of peripheral 
neuropathy can range from numbness in distal extremities, which makes fine motor activities challenging, to pain or difficulty with ambulation. Comorbidities for the neuropathy should be considered, and treatment often involves drugs that affect the central nervous system, such as gabapentin, amitriptyline, pregabalin, and duloxetine. Treatment efficacy data in the literature are mixed, and further large-scale randomized controlled studies are needed. ${ }^{18}$

Menopausal symptoms These symptoms are associated with ovarian failure related to treatment and use of endocrine therapy such as selective estrogen receptor modulators (SERMs) or aromatase inhibitors. ${ }^{14}$ Some younger, premenopausal women may have undergone ovarian ablation as part of their treatment regimen. Symptoms may include hot flashes, vaginal dryness, dyspareunia, and sleep disturbances. Some women, especially those under age 40 years, may have a return of ovarian function within 2 years of completing treatment, but can report poor self-image or sexual issues. Casey and colleagues suggest that these women should be asked about their reproductive life plan and be offered appropriate contraceptive options. ${ }^{19}$ For women who need contraception options during and after breast cancer treatment, the US medical eligibility criteria for contraceptive use is the reference of choice. ${ }^{20}$

Vasomotor symptoms are the most commonly reported symptom among menopausal women, and breast cancer survivors are no different. Because estrogen therapy is contraindicated, nonhormonal options can be considered. ${ }^{21}$ First-line interventions include avoiding triggers such as caffeine, alcohol, and spicy foods; dressing in layers of cool clothing; yoga, exercise, soy, relaxation therapy, and acupuncture also may help. ${ }^{22}$ The current consensus is that soy foods are safe for breast cancer survivors. ${ }^{23}$ In addition, pharmacotherapy with selective serotonin reuptake inhibitors (SSRIs), serotonin-norepinenephrine reuptake inhibitors (SNRIs), gabapentin, or clonidine shows mild to moderate improvement in vasomotor symptoms in randomized control trials. ${ }^{22}$ However, caution is advised with SSRIs, as they may interact with and reduce the effectiveness of tamoxifen. ${ }^{24}$

Vulvovaginal atrophy can be particularly difficult for women on long-term aromatase inhibitor therapy, and quality-of-life issues such as dyspareunia or dysuria can affect medication compliance. ${ }^{25}$ Estrogen supplementation is the ideal remedy but is generally contraindicated in this population. A trial of vaginal moisturizers and lubricants is best, but if symptoms are severe, estrogen supplementation via ring, cream, or tablet form, using products with the least systemic absorption, may be discussed.

Cognitive changes After initial treatment, $16 \%$ to $71 \%$ of patients develop cognitive changes such as changes in memory, mental cloudiness, difficulty in concentration,
TABLE 1. Advice for post-treatment breast examinations

- Examine the patient in the seated and supine positions. Remove clothing on the trunk and arms.

- View entire anterior chest while standing in front of the patient to assess symmetry, contour, and skin color. The port site incision may be seen just inferior to the clavicle.

- Ask the patient to lift her arms to 90 degrees. Watch for breast dimpling or nipple inversion and assess each arm for lymphedema, which may be found in the upper arm only, or entire arm and hand.

- Proceed with the usual careful breast examination.

- Complete the supraclavicular, infraclavicular, and axillary node examinations.

- The lumpectomy site should have a well-healed incision. The patient may have fibrosis secondary to radiation. Document this and show the patient how to check this area for nodules; ask for a return demonstration.

- In patients who are postmastectomy without reconstruction, sweep across the chest wall with a light-to-moderate touch, covering the entire area from clavicle to breast, including the axillae (Figure 1).

- In patients who are postmastectomy with reconstruction, perform the usual breast examination. No imaging is necessary unless surgery was nipple-sparing, as with some prophylactic surgery.

- If the nonaffected breast has had reduction mammoplasty, note incision lines, and complete the usual breast examination (Figure 2).

and significant reductions in multitasking ability or speed. ${ }^{26,27}$ These changes are more prevalent with increased age and may be associated with depression or anxiety. ${ }^{14}$ Refer the patient for cognitive therapy or stimulants if symptoms are severe.

Secondary malignancies Myelodysplasia or acute myelogenous leukemia are a recognized late effect from anthracyclines and alkylating chemotherapeutic agents, particularly when given in high doses. Risk is highest in the 5 to 10 years following treatment, and overall incidence is less than $5 \% .{ }^{28,29}$ Radiation treatment for breast cancer may increase the risk of secondary malignancies such as lung or esophageal cancers, but the effect is typically seen 10 years or more after treatment ends. Incidence appears related to total dose, earlier age at treatment, and genetic risk factors. ${ }^{29}$

Reduced bone mineral density Ovarian failure related to treatment or prolonged exposure to endocrine therapy can reduce bone mineral density. ${ }^{19}$ Estrogen supplementation is not appropriate treatment because it could stimulate breast cancer. However, other typical treatments, such as calcium, vitamin D supplementation, weight-bearing exercise, smoking cessation, and bisphosphonates, are warranted per the current standard of 
care for osteoporosis. Bone density scans are recommended every 12 to 24 months to assess patients for bone density changes related to aromatase inhibitor therapy. ${ }^{19}$

Upper extremity lymphedema Surgical removal of axillary lymph nodes can lead to upper extremity lymphedema at any time after surgery-typically within 3 years, although it can occur years later. ${ }^{14,30,31}$ Overall incidence ranges from $10 \%$ to $50 \%$ in women who underwent complete axillary lymph node dissection to $5 \%$ to $20 \%$ in women who underwent more limited sentinel lymph node biopsy. ${ }^{31}$ The risk is higher the more lymph nodes were removed, and in patients who had mastectomy or radiation therapy. Potential triggers can include constriction (such as with a BP cuff), insect bites, venipuncture, local trauma, prolonged (more than 3 hours) air travel in pressurized cabins, or repetitive arm motions. Air travel as a trigger for lymphedema has not been well studied, though a recent small study showed that for most women, air travel did not adversely affect the interlimb impedance

\section{TABLE 2. Resources for professional and patient support}

\section{For professionals:}

- Breast cancer follow-up and management after primary treatment: American Society of Clinical Oncology clinical practice guideline update. www.asco.org/guidelines/ breastfollowup.

- American Society of Clinical Oncology (ASCO). Cancer survivorship. www.asco.org/advocacy-policy/asco-inaction/asco-cancer-survivorship-compendium-providespractical-practice-tools.

- National Comprehensive Cancer Network. NCCN clinical practice guidelines in oncology: breast cancer screening and diagnosis, version 1.2016. https://www.nccn.org/ professionals/physician_gls/pdf/breast-screening.pdf.

- NCCN guidelines insights-survivorship, version 1.2016: https://education.nccn.org/node/78599.

For patients:

- American Cancer Society. Survivorship: during and after treatment. www.cancer.org/treatment/survivorship duringandaftertreatment/.

- ASCO. Follow-up care for breast cancer. www.cancer.net/ publications-and-resources/what-know-ascos-guidelines/ what-know-ascos-guideline-follow-care-breast-cancer/ recommendations.

- ASCO care and treatment recommendations for patients. www.cancer.net/research-and-advocacy/asco-care-andtreatment-recommendations-patients.

- Sisters Network, Inc.: A national African-American breast cancer survivor organization. www.sistersnetworkinc.org/.

- Susan G. Komen. Life after treatment. http://ww5.komen. org/LifeAfterTreatment.html. ratio. ${ }^{32}$ The traditional list of triggers also includes heavy lifting, although evidence suggests that progressive resistance exercise therapy does not increase the risk of developing lymphedema and may actually help lymph system drainage. ${ }^{33}$

Refer patients with lymphedema to physical therapy for decongestive therapy and manual lymphatic drainage. Patients can be trained to avoid the triggers and selfmanage symptoms with compressive garments and devices. ${ }^{30}$ At present, evidence is insufficient to support or refute the clinical recommendations to wear compression garments during regular exercise. However, when compression garments are worn, they should be measured to the patient's arm size and worn before repetitive motion activities or during extended air travel..$^{34}$ Signs of cutaneous infections in affected limbs should be treated promptly with antibiotic coverage for Staphylococcus and Streptococcus. Women with recurrent cellulitis may need daily antibiotic therapy.

Reproductive considerations Between 15\% and 25\% of breast cancer cases occur in premenopausal women, and $7 \%$ are diagnosed in women under age 40 years, so reproductive issues are an important part of cancer treatment planning. ${ }^{25}$ Women who wish to become pregnant after treatment should discuss this issue before surgery, chemotherapy, or endocrine therapy, as each may directly affect fertility, lactation, or timing of pregnancy attempts. Data now suggest that women can achieve a healthy pregnancy after breast cancer treatment, without negatively affecting their disease-free or overall survival, regardless of hormone receptor status. ${ }^{19,35}$ For premenopausal women who do not desire future pregnancy, hormone-free contraception options include permanent sterilization (male or female), copper intrauterine devices, or barrier methods. Amenorrhea and elevated follicle-stimulating hormone levels are unreliable indicators of infertility in women who have received chemotherapy. As many as $53 \%$ to $89 \%$ of women will become amenorrheic during chemotherapy; this is more likely to be reversible in women under age 40 years. ${ }^{19}$

\section{LONG-TERM ENDOCRINE THERAPIES}

Many breast cancer survivors who had a hormone receptor-positive cancer will receive up to 10 years of endocrine therapy to decrease their risk of cancer recurrence. ${ }^{36}$ The oncologist most commonly uses tamoxifen or an aromatase inhibitor (letrozole, exemestane, or anastrozole), depending on the patient's menopausal status. Studies show a $46 \%$ to $58 \%$ reduction in new primary contralateral breast cancers with these therapies. ${ }^{37}$

In premenopausal women, tamoxifen is the favored antiestrogen medication and was given for a total of 5 years (more recently, for 10 years). ${ }^{36,37}$ Potential adverse reactions include vasomotor symptoms, increased risk of venous thrombosis and cataracts, and a slightly 
increased risk of endometrial cancer. ${ }^{38}$ Depending on the patient's initial cancer stage and tumor characteristics, as well as menopausal status, an additional 5 years of aromatase inhibitor therapy may be recommended after completion of tamoxifen. Aromatase inhibitors are used in postmenopausal patients and reduce circulating estrogen levels by inactivating the aromatase enzyme required to convert androstenedione to estrone and estradiol in breast, muscle, and fat tissue. Adverse reactions may include loss of bone mineral density; some patients receive IV bisphosphonates adjuvantly to prevent bone loss. ${ }^{39}$

Endocrine therapies also can cause a slightly increased cardiovascular risk, arthralgia, and vasomotor symptoms.

For more evidence-based information on managing breast cancer survivors, as well as patient-focused survivor support, see Table 2 .

\section{CONCLUSION}

Breast cancer survivors are transitioning to primary care providers earlier after acute treatment and in larger numbers than in years past. Primary care providers need to know key surveillance, history, and physical examination techniques, as well as the long-term and late effects of treatment. Providers should pay special attention to cardiac symptoms, menopausal symptoms, quality of life, and a lymphedema survey. National evidence-based guidelines can help primary care providers deliver optimal care to breast cancer survivors. JAAPA

Earn Category I CME Credit by reading both CME articles in this issue, reviewing the post-test, then taking the online test at http://cme.aapa. org. Successful completion is defined as a cumulative score of at least $70 \%$ correct. This material has been reviewed and is approved for 1 hour of clinical Category I (Preapproved) CME credit by the AAPA. The term of approval is for 1 year from the publication date of October 2016.

\section{REFERENCES}

1. American Cancer Society. Breast cancer facts \& figures 20152064.www.cancer.org/acs/groups/content/@research/documents/ document/acspc-046381.pdf. Accessed August 1, 2016.

2. American Cancer Society. Cancer treatment facts $\&$ figures 2016-2017.www.cancer.org/acs/groups/content/@research/ documents/document/acspc-048074.pdf. Accessed August 1, 2016.

3. Grant M, De Rossi S, Sussman J. Supporting models to transition breast cancer survivors to primary care: formative evaluation of a cancer care Ontario initiative. J Oncol Pract. 2015;11(3):e288e295.

4. Erikson C, Salsberg E, Forte G, et al. Future supply and demand for oncologists: challenges to assuring access to oncology services. J Oncol Pract. 2007;3(2):79-86.

5. Kantsiper M, McDonald EL, Geller G, et al. Transitioning to breast cancer survivorship: perspectives of patients, cancer specialists, and primary care providers. J Gen Intern Med. 2009;24(suppl 2):S459S466.

6. Maly RC, Liu Y, Diamant AL, Thind A. The impact of primary care physicians on follow-up care of underserved breast cancer survivors. J Am Board Fam Med. 2013;26(6): 628-636.
7. National Comprehensive Cancer Network. NCCN clinical practice guidelines in oncology: breast cancer, version 2.2016. https://www. nccn.org/professionals/physician_gls/pdf/breast.pdf. Accessed July 29, 2016.

8. Khatcheressian JL, Hurley P, Bantug E, et al. Breast cancer follow-up and management after primary treatment: American Society of Clinical Oncology clinical practice guideline update. J Clin Oncol. 2013;31(7):961-965.

9. National Comprehensive Cancer Network. NCCN clinical practice guidelines in oncology: breast cancer screening and diagnosis, version 1.2016. Accessed July 29, 2016. https://www. nccn.org/professionals/physician_gls/pdf/breast-screening.pdf.

10. Moyer VA; U.S. Preventive Services Task Force. Risk assessment, genetic counseling, and genetic testing for BRCA-related cancer in women: U.S. Preventive Services Task Force recommendation statement. Ann Intern Med. 2014;160(4):271-281.

11. O’Sullivan B, Levin W. Late radiation-related fibrosis: pathogenesis, manifestations, and current management. Semin Radiat Oncol. 2003;13(3):274-289.

12. Srivastava V, Basu S, Shukla VK. Seroma formation after breast cancer surgery: what we have learned in the last two decades. J Breast Cancer. 2012;15(4):373-380.

13. Hewitt M, Greenfield S, Stovall E, eds. From Cancer Patient to Cancer Survivor: Lost in Transition. Washington, DC: The National Academies Press; 2005.

14. Kenyon M, Mayer DK, Owens AK. Late and long-term effects of breast cancer treatment and surveillance management for the general practitioner. J Obstet Gynecol Neonatal Nurs. 2014;43(3): 382-398.

15. Siegel R, DeSantis C, Virgo K, et al. Cancer treatment and survivorship statistics, 2012. CA Cancer J Clin. 2012;62(4): 220-241.

16. Simone NL, Dan T, Shih J, et al. Twenty-five year results of the National Cancer Institute randomized breast conservation trial. Breast Cancer Res Treat. 2012;132(1):197-203.

17. Stubblefield MD, McNeely ML, Alfano CM, Mayer DK. A prospective surveillance model for physical rehabilitation of women with breast cancer: chemotherapy-induced peripheral neuropathy. Cancer. 2012;118(8 suppl):2250-2260.

18. Hershman DL, Lacchetti C, Dworkin RH, et al. Prevention and management of chemotherapy-induced peripheral neuropathy in survivors of adult cancers: American Society of Clinical Oncology clinical practice guideline. J Clin Oncol. 2014;32(18):1941-1967.

19. Casey PM, Faubion SS, MacLaughlin KL, et al. Caring for the breast cancer survivor's health and well-being. World J Clin Oncol. 2014;5(4):693-704.

20. Centers for Disease Control and Prevention. US medical eligibility criteria for contraceptive use, 2010. Adapted from the World Health Organization medical eligibility criteria for contraceptive use, 4th edition. MMWR. 2010;59(RR-4):1-86.

21. Chlebowski RT, Anderson GL. Changing concepts: menopausal hormone therapy and breast cancer. J Natl Cancer Inst. 2012; 104(7):517-527.

22. Rada G, Capurro D, Pantoja T, et al. Non-hormonal interventions for hot flushes in women with a history of breast cancer. Cochrane Database Syst Rev. 2010;(9):CD004923.

23. Dong JY, Qin LQ. Soy isoflavones consumption and risk of breast cancer incidence or recurrence: a meta-analysis of prospective studies. Breast Cancer Res Treat. 2011;125(2):315-323.

24. Brauch H, Schroth W, Goetz MP, et al. Tamoxifen use in postmenopausal breast cancer: CYP2D6 matters. J Clin Oncol. 2013;31(2):176-180.

25. Moegele M, Buchholz S, Seitz S, Ortmann O. Vaginal estrogen therapy in postmenopausal breast cancer patients treated with aromatase inhibitors. Arch Gynecol Obstet. 2012;285(5): 1397-1402. 
26. Evens K, Eschiti VS. Cognitive effects of cancer treatment: "chemo brain" explained. Clin J Oncol Nurs. 2009;13(6): 661-666.

27. Tager FA, McKinley PS, Schnabel FR, et al. The cognitive effects of chemotherapy in post-menopausal breast cancer patients: a controlled longitudinal study. Breast Cancer Res Treat. 2010; 123(1):25-34.

28. Azim HA Jr, de Azambuja E, Colozza M, et al. Long-term toxic effects of adjuvant chemotherapy in breast cancer. Ann Oncol. 2011;22(9):1939-1947.

29. Bodai BI, Tuso P. Breast cancer survivorship: a comprehensive review of long-term medical issues and lifestyle recommendations. Perm J. 2015;19(2):48-79.

30. Fu MR. Breast cancer-related lymphedema: symptoms, diagnosis, risk reduction, and management. World J Clin Oncol. 2014;5(3): 241-247.

31. Loh SY, Musa AN. Methods to improve rehabilitation of patients following breast cancer surgery: a review of systematic reviews. Breast Cancer (Dove Med Press). 2015;7:81-98.

32. Kilbreath SL, Ward LC, Lane K, et al. Effect of air travel on lymphedema risk in women with history of breast cancer. Breast Cancer Res Treat. 2010;120(3):649-654.

33. Stuiver MM, ten Tusscher MR, Agasi-Idenburg CS, et al. Conservative interventions for preventing clinically detectable upperlimb lymphoedema in patients who are at risk of developing lymphoedema after breast cancer therapy. Cochrane Database Syst Rev. 2015;(2):CD009765.

34. Singh B, Disipio T, Peake J, Hayes SC. Systematic review and meta-analysis of the effects of exercise for those with cancerrelated lymphedema. Arch Phys Med Rehabil. 2016;97(2): 302-315.e13.

35. de Pedro M, Otero B, Martín B. Fertility preservation and breast cancer: a review. Ecancermedicalscience. 2015;9:503.

36. Burstein HJ, Temin S, Anderson H, et al. Adjuvant endocrine therapy for women with hormone receptor-positive breast cancer: American Society of Clinical Oncology clinical practice guideline focused update. J Clin Oncol. 2014;32(21):2255-2269.

37. Birru M, Sanfilippo K, Vogel VG. Primary prevention of breast cancer In: Bland KI, Copeland EM III, eds. The Breast: Comprehensive Management of Benign and Malignant Disease. 4th ed. Philadelphia, PA: Saunders; 2009:349-370.

38. Davies C, Pan H, Godwin J, et al. Long-term effects of continuing adjuvant tamoxifen to 10 years versus stopping at 5 years after diagnosis of oestrogen receptor-positive breast cancer: ATLAS, a randomised trial. Lancet. 2013;381(9869): 805-816.

39. Brufsky AM, Harker WG, Beck JT, et al. Final 5-year results of Z-FAST trial: adjuvant zoledronic acid maintains bone mass in postmenopausal breast cancer patients receiving letrozole. Cancer. 2012;118(5):1192-1201. 